\title{
Rilievo e modellazione digitale: un percorso critico per la valorizzazione del Castello di Ischia
}

Survey and digital modeling: a critical approach for the enhancement of the Castle of Ischia

\author{
Lia Maria Papa ${ }^{\text {, }}$,Saverio D’Auria ${ }^{\text {b }}$ \\ Dipartimento di Ingegneria Civile, Architettonica e Ambientale - Università degli Studi di Napoli Federico II, Naples, Italy \\ almpapa@unina.it; ${ }^{\mathrm{b}}$ saverio.dauria@unina.it
}

\begin{abstract}
The development and dissemination of ICT have also influenced the cultural heritage sector. In the last decade, for this reason and not only, the way of doing scientific research, documentation and enhancement has quickly changed, to such a degree to question the real benefits brought by the digitization and virtualization of the historical buildings, in the fields just mentioned.

The paper is part of a research field linked to the analysis of the metric and formal reliability of 3D integrated surveying and digital modeling of architectural heritage, and to the critical evaluation of the photogrammetry and laser scanning as tools for the deep knowledge and for the dissemination of the object of study.

In particular, the focus is on the majestic Aragonese Castle of Ischia -whose origins date back to the fifth century BC, located in the eastern part of the island, on an area of over 5 hectares- and on the ruins of the Cathedral of the Assumption, of the fourteenth century, preserved inside.

Starting from particular analysis and coming to general methodological considerations, the research had different objectives: comparing qualitatively and quantitatively the goodness of image-based and range-based surveys of single environments, determining the spatial location of some volumes compared to others, analyzing the "Castle-Cathedral system" through the development of virtual reality applications built from photo-modeling and laser scanning at the urban scale.
\end{abstract}

Keywords: 3D surveying, virtual reality, cultural heritage, fortifications.

\section{Introduzione}

Il patrimonio culturale è oramai posto al centro di un processo virtuoso di conoscenza, valorizzazione, fruizione. Esso è testimonianza di vicende storiche ed eventi naturali che spesso hanno compromesso l'originaria configurazione dei luoghi e degli artefatti.

In tale processo un ruolo di supporto non trascurabile è offerto dalle nuove tecnologie digitali di acquisizione delle informazioni e di comunicazione.

Di fatto nell'ultimo decennio è mutato anche il modo di fare ricerca, divulgazione, documentazione e valorizzazione al punto tale da dover constatare talvolta una minore attenzione al processo conoscitivo nella sua più rigorosa connotazione e complessità a fronte della elaborazione di prodotti dalla forte caratterizzazione evocativa e percettiva.

Il contributo documenta uno studio appena intrapreso che si inserisce in un campo di ricerca 
più ampio legato all'analisi dell'affidabilità metrico-formale del rilevamento 3D integrato e della modellazione digitale del patrimonio architettonico, e alla valutazione critica degli apporti della fotogrammetria e del laser scanning alla conoscenza profonda e alla disseminazione dell'oggetto di studio.

L'oggetto di studio è un monumento dalla forte caratterizzazione costruttiva e valenza storica: il Castello Aragonese di Ischia (Fig. 1). Esso, in realtà, si configura come un complesso fortificato che occupa una superficie di oltre 5 ettari, in cui sono presenti anche testimonianze stratificate e resti di edifici di pregio, come in particolare quelli della Cattedrale dell' Assunta.

La ricerca avviata, partendo da analisi particolari e giungendo a considerazioni metodologiche generali, ha diversi obiettivi: confrontare qualitativamente e quantitativamente la bontà dei rilievi image e range-based (su supporto topografico), documentare l'architettura mediante i grafici di rilievo (spesso inediti), definire la collocazione spaziale di alcuni volumi rispetto ad altri, analizzare formalmente il 'sistema Castello', anche attraverso l'elaborazione di realtà virtuali alla scala urbana.

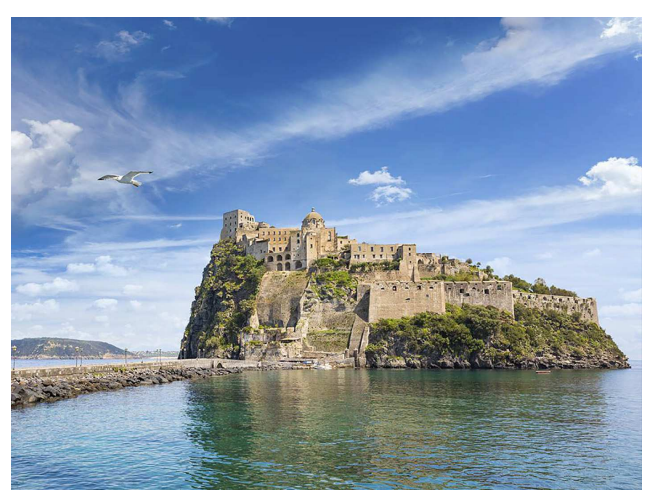

Fig. 1. Fotografia dell'isola e del Castello.

In particolare, il presente articolo descrive alcuni dei risultati ottenuti a seguito della campagna di rilievo integrato dei resti della Cattedrale dell'Assunta, effettuata successivamente alle indagini morfometriche e cromatiche condotte sulla sottostante cripta gentilizia, già oggetto di studio.

\section{Il contesto storico-architettonico}

Il complesso monumentale del castello, situato nella parte orientale dell'isola, risale all'inizio del V secolo a.C. Nel corso del tempo costituì un valido rifugio per la popolazione dalle invasioni barbariche e dalle scorrerie dei saraceni.

Alla fine del XII secolo esso perse definitivamente i caratteri esclusivi di fortificazione militare, trasformandosi in sede istituzionale dell'isola e residenza delle famiglie nobili. Nelle successive guerre tra Svevi e Angioini prima, e tra Angioini e Aragonesi poi, fu oggetto di distruzioni, ricostruzioni e ampliamenti. A seguito della colata lavica del 1301, gli Ischitani si rifugiarono nel castello e lo trasformano in una vera e propria cittadella erigendo, inoltre, la Cattedrale dell'Assunta in corrispondenza della cripta gentilizia risalente all'XI secolo.

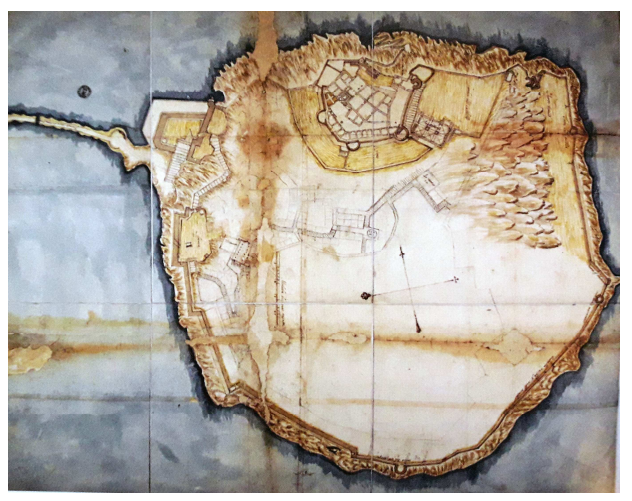

Fig. 2. Pianta del Castello di Ischia del XVI secolo (Tortelli B., Biblioteca Nazionale di Napoli, Ms. XII D).

Con il regno di Alfonso I d'Aragona il manufatto assunse la doppia funzione di roccaforte e residenza reale. Nel XVII secolo gli Spagnoli riconquistarono Ischia e resero funzionanti solo pochi edifici pubblici come il maschio, adibito a fortezza militare e a carcere, la cattedrale, il palazzo vescovile e il convento (Fig. 2). Nella veduta settecentesca di Figura 3 è evidente la connotazione vulcanica dell'isola con la presenza del castello e delle case arroccate sul masso trachitico.

In seguito alla restaurazione borbonica, il complesso divenne carcere politico che fu soppresso 
nel 1860 quando Ischia fu annessa al Regno d'Italia.

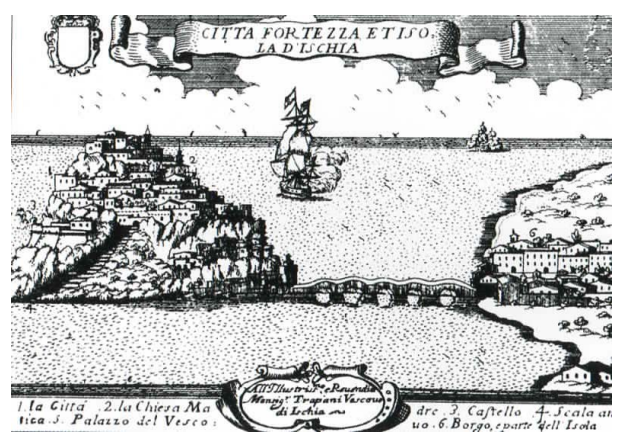

Fig. 3. Città e fortezza et Isola d'Ischia, (Francesco Cassiano de Silva, attr., 1703. Biblioteca Nazionale di Napoli, Sezione Manoscritti e Rari, SQ. XXXIV B19).

Un testo fondamentale per lo studio storicoartistico dell'isola e delle sue emergenze architettoniche è il manoscritto redatto dal canonico ischitano Vincenzo Onorato, dal titolo Ragguaglio istorico topografico dell'Isola d'Ischia ${ }^{1}$, che compensa l'assenza della quasi totalità dei documenti antichi andati perduti per le gravi vicende subite in più riprese dall'Archivio Diocesano dell'isola ${ }^{2}$.

Il religioso, infatti, è stato l'ultimo a descrivere la Cattedrale dell'Assunta (e la sottostante cripta gentilizia) prima dei tremendi bombardamenti del 1809 a cui fu sottoposto il Castello dalle truppe anglo-borboniche nel tentativo, riuscito, di liberare l'isola dal dominio napoleonico.

Quegli eventi portano alla distruzione dell'edificio sacro, conservato oggi a stato di rudere nella stessa configurazione di due secoli fa (Fig. 4).

Nel Ragguaglio si legge che: "L'antica chiesa Cattedrale vescovile [la cripta gentilizia, ndr] stava sita nel recinto del Castello, e riguardava soprattutto il sud [...]. La medesima Cattedrale era una semplice cappella lunghetta sì, ma stretta ed all'infuori dell'altare maggiore non ci era altro altare. $[\ldots]$ La nuova Cattedrale [dell'Assunta, ndr], opera del $12^{\circ}$ secolo, fu edificata al di sopra della prefata ed in modo che essa divenne soccorpo della nuova, ma solo in una tirata di laterale che guardava il sud, e di maniera che la porta maggiore porgeva quasi a perpendicolo su la porta maggiore della vecchia.

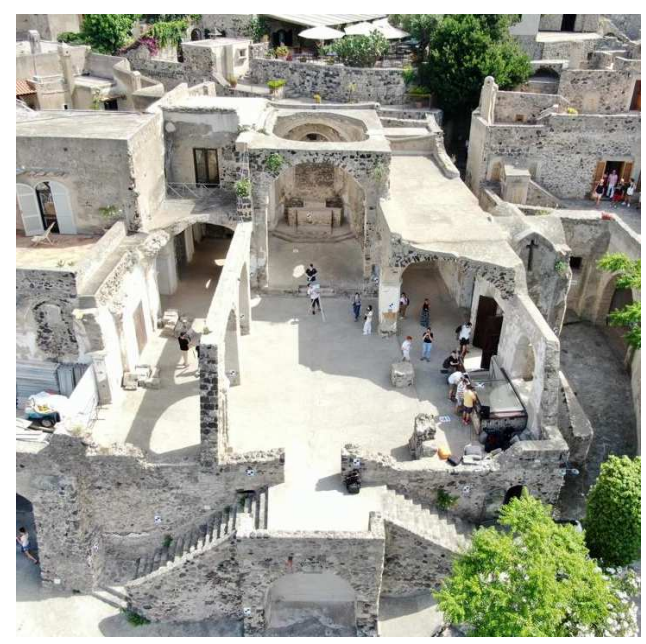

Fig. 4. Vista aerea dei resti della Cattedrale dell'Assunta durante la campagna di rilevamento (foto Chianese D.).

La costruzione fu all'opposto dell'antica, per essersi formata a tre navate e colle cappelle laterali e così larga e lunga che nella magnificenza nulla era proporzionata col Castello. Di sette cappelle sei erano gentilizie [...]".

\section{Il rilievo sistematico e integrato del sistema cripta-Cattedrale}

Il progetto di rilevamento tridimensionale dei resti della Cattedrale dell'Assunta e della sottostante cripta gentilizia ha contemplato l'impiego di più metodi di acquisizione dei dati metrici e cromatici, integrandoli opportunamente.

In particolare: il laser-scanning (che si è avvalso di uno Z+F Imager 2010C) è stato impiegato per rilevare quelli che anticamente erano gli ambienti interni della Cattedrale, nonché gli spazi immediatamente esterni, sia per definire correttamente i volumi sia per circoscrivere l'intorno urbano; l'aerofotogrammetria da drone (mediante un DJI Phantom 2 Plus e un DJI Mavic 2 zoom) si è resa necessaria per il rilievo delle coperture e del contesto antropizzato in cui si colloca l'architettura studiata; la fotogrammetria terrestre (facendo uso di smartphone e di reflex semi-professionali quali una Canon EOS 750D e una Nikon D3100) è stata impiegata per l'analisi delle superfici decorate; il rilievo topografico (con stazione totale Nikon NPL-352 e un ricevitore GPS Topcon Hiper Pro), invece, è stato di 
fondamentale supporto per georiferire le nuvole di punti, per controllare i processi di fotomodellazione e, soprattutto, per collocare spazialmente in maniera corretta i modelli 3D della cripta gentilizia e della sovrastante Cattedrale (Fig. 5).

Per il rilevamento mediante laser scanner sono stati previsti ed effettuati 34 stazionamenti (Fig. 6 ), seguendo un percorso che, in senso antiorario, partiva dall'esterno della facciata principale a sud (ora inesistente), proseguiva lungo i percorsi che costeggiano il fabbricato ed entrava nei resti della Cattedrale, compresa la sagrestia (oggi intatta).

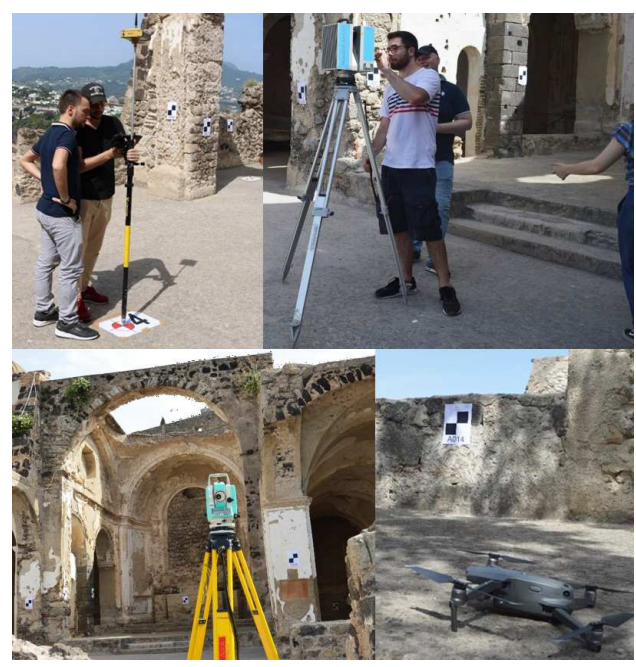

Fig. 5. Alcune delle strumentazioni impiegate per l'acquisizione delle informazioni metriche.

La risoluzione delle scansioni è stata mantenuta alta (un punto battuto ogni $15 \mathrm{~mm}$ circa a distanza di $10 \mathrm{~m}$ ) per gli spazi esterni e media (un punto battuto ogni $30 \mathrm{~mm}$ circa a distanza di $10 \mathrm{~m}$ ) per gli ambienti volumetricamente chiusi e di dimensioni contenute (sagrestia e cappelle laterali).

Contestualmente, è stato eseguito il rilievo con stazione totale e con ricevitore GPS: in particolare, la stazione totale è stata di fondamentale supporto per il collegamento "interno-esterno" della cripta ipogea (già oggetto di rilievo fotogrammetrico $^{3}$ ) e per la definizione delle coordinate di alcuni punti ritenuti importanti per le successive valutazionimorfo-metriche (sono sta- ti battuti 38 punti); mentre il GPS ha individuato a terra, con la materializzazione di target ad alto contrasto cromatico in formato cartaceo $\mathrm{A} 3$, le coordinate di 10 punti, implementati poi nei processi di fotomodellazione in qualità di GCPs.

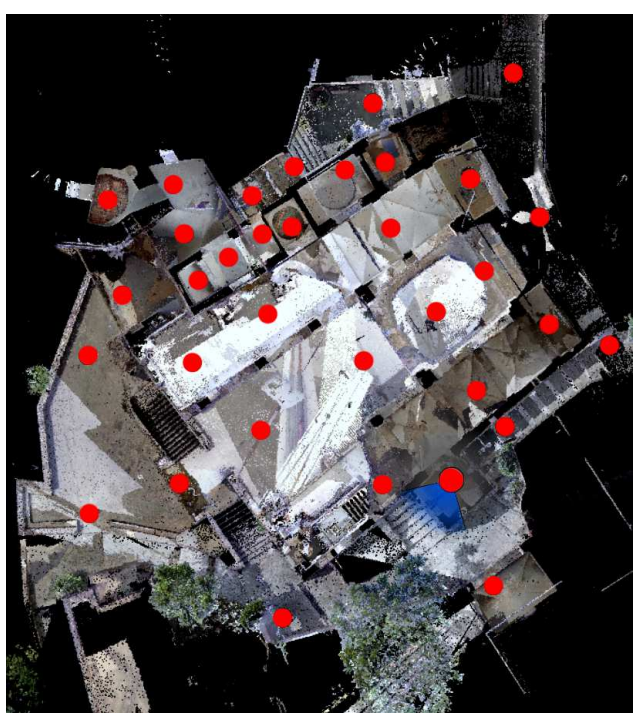

Fig. 6. I punti di stazionamento laser.

L'aerofotogrammetria, infine, si è avvalsa di due droni equipaggiati con camere differenti una da 14 (Phantom) l'altra da 12 (Mavic) megapixel. Le prese fotografiche sono state effettuate ad una quota di volo di circa $20 \mathrm{~m}$ dal piano medio della navata centrale, garantendo, come di consueto, una superficie di sovrapposizione tra scatti consecutivi di almeno il 70\%; gli assi delle camere sono stati mantenuti zenitali per le strisciate sulle coperture effettuate con piani di volo assistiti a doppia griglia, mentre a $45^{\circ}$ per i voli in modalità ATTI con traiettorie circolari intorno alla fabbrica (Fig. 7).

Sono state scattate in totale 278 foto (109 con il primo drone, 169 con il secondo) utili per i processi di fotomodellazione. I dati ottenuti dalle campagne di rilevamento (nuvole di punti, fotografie, matrici numeriche) sono stati elaborati nelle successive fasi di post-processamento attraverso l'uso di macchine mediamente performanti, software diffusi e affidabili e procedure ormai consolidate da anni nell'ambito della disciplina. La nuvola di punti da laser scanning 
(Fig. 8) conta quasi 260 milioni di punti con un errore medio di allineamento non superiore ai 4 $\mathrm{mm}$ e pesa circa 20 gigabyte; il modello imagebased (Fig. 9) al momento prodotto -quello relativo alle foto da 12 megapixel- conta quasi 172 milioni di punti, con errori di allineamento inferiori al pixel alla massima qualità di elaborazione e ha una dimensione di circa 7 GB; risulta, inoltre, valido dal punto di vista formale e metrico grazie all'implementazione dei punti di controllo (GPS e stazione totale). Gli stessi hanno consentito di integrare i tre modelli differenti rappresentativi: delle coperture e del contesto urbano (da fotogrammetria aerea), della Cattedrale e del suo intorno (da laser-scanning) e della cripta (da fotogrammetria terrestre).

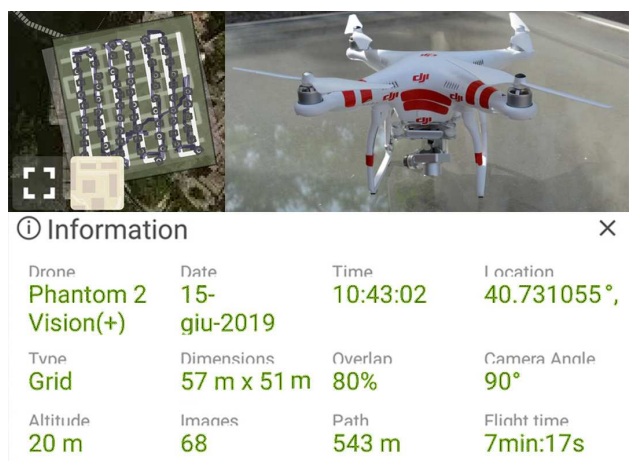

Fig. 7. Uno dei piani di volo.

\section{Risultati e conclusioni}

Il presente contributo mostra i primi risultati di una più ampia ricerca in corso sul Castello di Ischia e sul patrimonio storico-edilizio in esso ospitato, volta ad analizzare da più punti di vista (morfologico, costruttivo, urbanistico-evolutivo, fruizionale) il contesto architettonico ad oggi presente.

Nello specifico, l'attenzione è stata rivolta ai resti della Cattedrale dell'Assunta e alla sottostante cripta gentilizia, già documentata in altro contributo. Per tali opere è stata effettuata, in maniera sistematica e scientificamente controllata, la campagna di rilevamento tridimensionale e la successiva fase di elaborazione dei dati acquisiti.
È in fase avanzata la redazione gli elaborati di rilievo alla scala di contesto e alla scala architettonica, andando ad aggiornare e, in alcuni casi, a editare per la prima volta la documentazione grafica.

La ricerca, ancora in corso, integrando criticamente i rilievi aggiornati e gli studi condotti da autorevoli storici del presente e del passato (seppur non numerosissimi, per la penuria di documenti già denunciata), consentirà inoltre una approfondita analisi delle trasformazioni costruttive subite dalla Cattedrale, dal medioevo agli inizi dell'Ottocento, proponendo grafici e ricostruzioni volumetriche in realtà virtuale, utili anche per scopi di valorizzazione turistico-culturale.

Non c'è dubbio che l'utilizzo consapevole delle più aggiornate tecnologie per il rilievo dell'architettura e del territorio e l'attenta valutazione delle informazioni acquisite, porti a nuove possibilità di lettura critica del costruito storico, con un conseguente avanzamento delle conoscenze negli ambiti di applicazione e ricadute sulle possibilità di disseminazione, in relazione al valore e significato storico-architettonico del monumento oggetto di studio.

\section{Note}

${ }^{1}$ Il manoscritto è conservato presso la Biblioteca Nazionale di Napoli, al n. 439 del Fondo San Martino. L'analisi e la trascrizione del documento è ad opera della storica Ernesta Mazzella.

${ }^{2}$ Sono pochi i documenti storici sul Castello di Ischia, in generale, e sulla Cattedrale dell'Assunta, in particolare. Alcuni disastrosi eventi, come l'eruzione dell' Arso di inizio Trecento, l'incendio durante la pestilenza del 1656 e l'occupazione dei soldati francesi ai primi dell'Ottocento, hanno distrutto gran parte delle testimonianze archivistiche presenti sull'isola.

${ }^{3}$ Le prese fotogrammetriche della cripta gentilizia sono state effettuate da Valeria Schiano Di Zipaolo nell'ambito della Tesi di Laurea in Rilievo e Progetto (novembre 2018) del Corso di Studi in Ingegneria Edile dell'Università degli Studi di Napoli Federico II. 


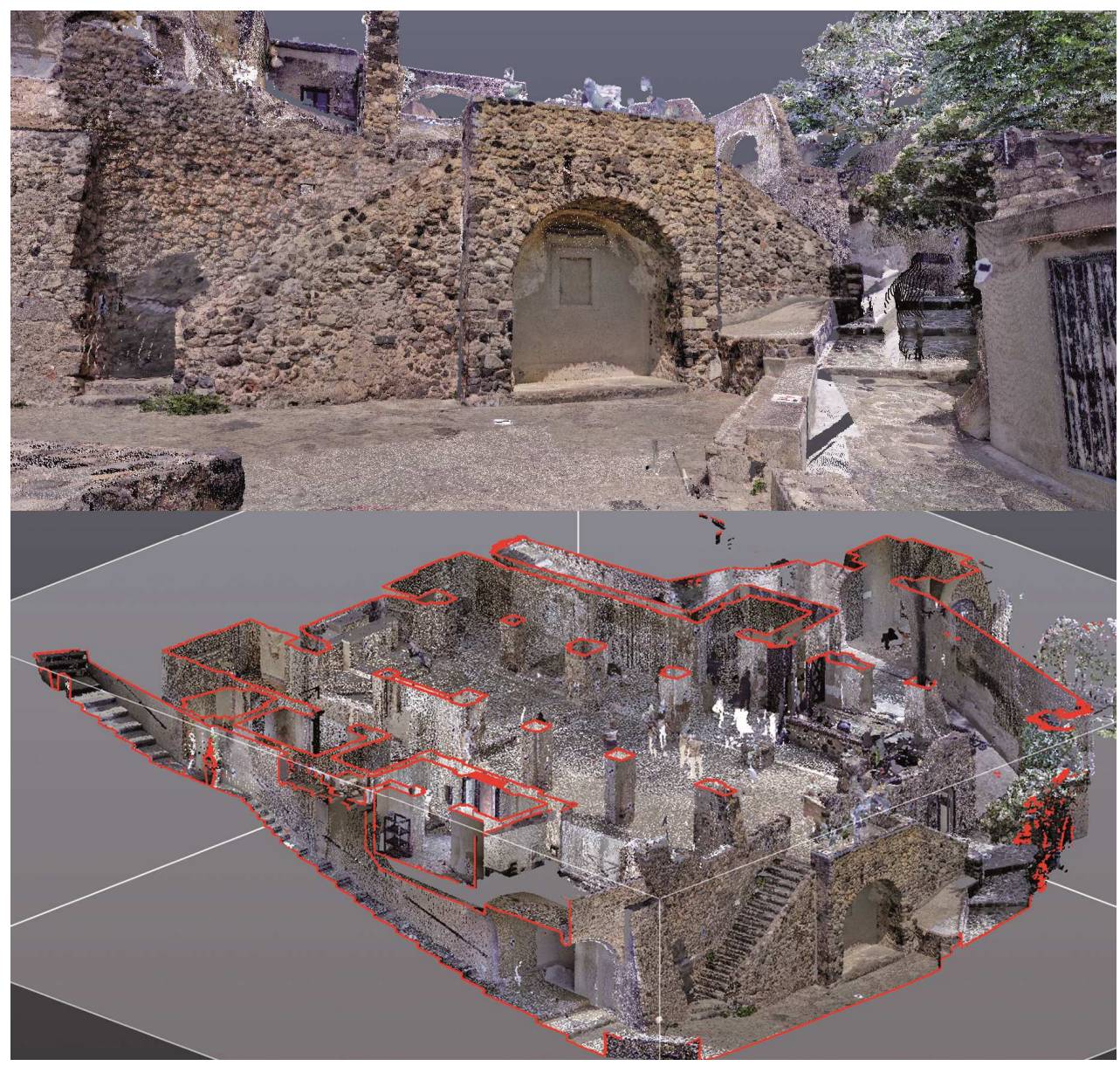

Fig. 8. Singola scansione laser dei resti della facciata principale rivolta a sud (in alto); modello da laser scanning della Cattedrale e dell'intorno, tranciato con un piano orizzontale e uno verticale (in basso).

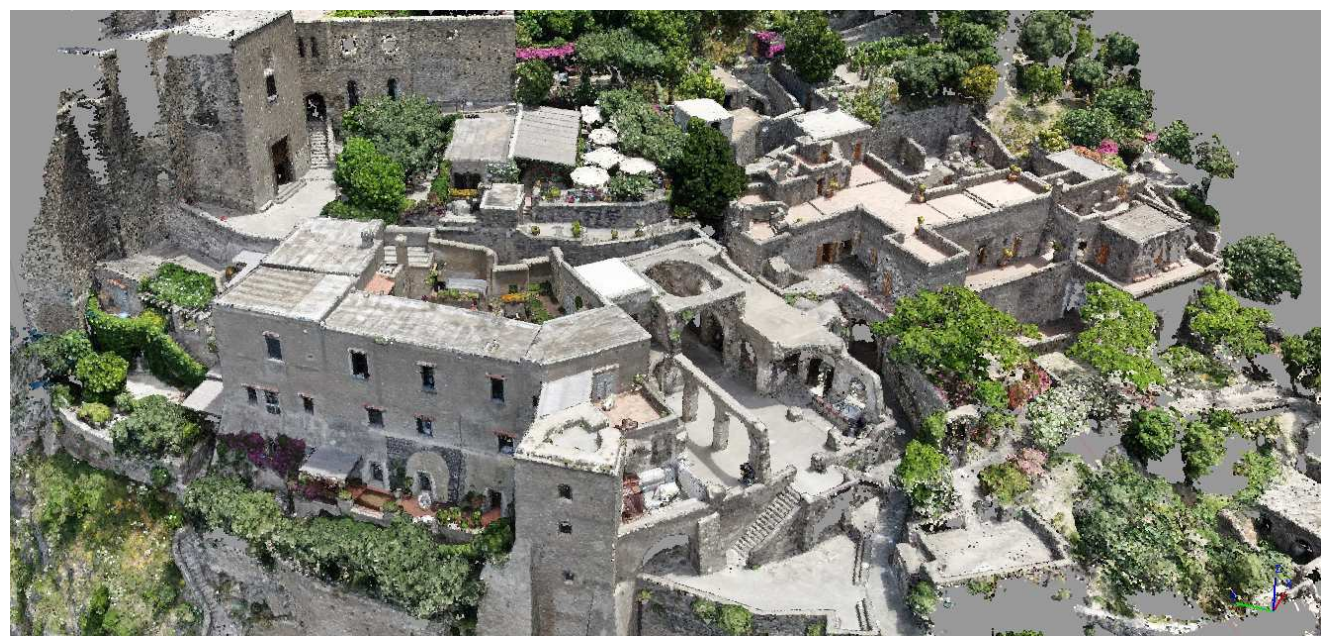

Fig. 9. Nuvola di punti image-based del contesto in cui attualmente sono collocati i resti della Cattedrale. 
L.M. Papa ha coordinato l'impianto complessivo del lavoro ed è autrice dei paragrafi 1 e 4.

S. D'Auria ha coordinato le attività di rilevamento sul campo ed è autore dei paragrafi 2 e 3.

Gli autori ringraziano l'architetto Nicola Mattera per la sua disponibilità a consentire l'accesso e le operazioni di rilevamento strumentale negli spazi del Castello interessati da questo studio, nonché Valeria Schiano Di Zipaolo, Valentina Curci, Emanuela De Feo e Domenico Chianese che, a vario titolo, hanno collaborato alla ricerca.
Il presente lavoro, inoltre, ha visto coinvolti diversi tesisti nell'ambito del corso di Rilievo e Progetto del corso di laurea in Ingegneria Edile: Miriana Annecchiarico, Carlo D'Aniello, Claudia Delle Cave, Immacolata Evidente, Armando Franzese, Alessandro Paolella, Flavia Stara e Maria Terracciano; alcuni di loro (Emanuele Caruso, Riccardo De Ieso e Matteo Iacobbi) hanno anche messo a disposizione le strumentazioni impiegate: laser scanner, GPS, stazione totale e drone, addivenendo ad una fertile esperienza di confronto laboratoriale.

\section{Bibliography}

Barba, S. (2017). "Rilievi e documentazione per la valorizzazione culturale della provincia di Mpumalanga (Sudafrica)", in Programmi multidisciplinari per l'internazionalizzazione della ricerca. Patrimonio culturale, Architettura e Paesaggio, Firenze didapress, pp. 203-208.

Curci, V. (2018). Committenza artistica a Ischia in etá angioina, Tesi di laurea magistrale in Archeologia e Storia dell'Arte, Dipartimento di Studi Umanistici dell'Università degli Studi di Napoli Federico II, Napoli.

Delizia, I. (1989). "Un'antica pianta del Castello Aragonese”, La rassegna d'Ischia, 10, pp. 28-31.

Farella, E.; Menna, F.; Remondino, F.; Campi, M. (2016). "3d modeling and virtual applications for the valorization of historical heritage", in 8th International Congress on archaeology, computer graphics, cultural heritage and innovation, Universitat Politècnica de València Ed., Valencia, pp. 456-459.

Guidi, G.; Russo, M.; Angheleddu, D. (2014). "3D survey and virtual reconstruction of archaeological sites”, Digital Applications in Archaeology and Cultural Heritage, 1, pp. 55-69.

Luongo, L. (2010). "Dipinti murali nella cripta della Cattedrale dell'Assunta sul Castello Aragonese", La rassegna d'Ischia, 1, pp. 10-11.

Mazzella, E. (2014). L'anonimo. Vincenzo Onorato e il Ragguaglio dell'isola di Ischia, Gutemberg Ed., Fisciano.

Papa, L.M.; D’Auria, S. (2019). "Valutazioni sul processo conoscitivo di architetture ipogee: la Cripta della Cattedrale nel Castello di Ischia”, in Belardi, P. ed., Atti del $41^{\circ}$ convegno internazionale dei docenti delle discipline della rappresentazione 'Riflessioni: l'arte del disegno/il disegno dell'arte', Gangemi International Ed., Roma, pp. 873-880.

Romano, R. (2006). "Dipinti murali nella chiesa inferiore della Cattedrale del Castello di Ischia: una testimonianza poco conosciuta di devozione privata", in Abbate, F. ed., Ottant'anni di un Maestro: omaggio a Ferdinando Bologna, Paparo Ed., Pozzuoli, pp. 137-145. 
